\title{
Degenerate double solids as twistor spaces
}

\author{
NOBUHIRO HONDA
}

\begin{abstract}
We show that there exist twistor spaces over $3 \mathbf{C P}^{2}$, the connected sum of three complex projective planes, whose half-anticanonical system gives a double covering map onto $\mathbf{C P}^{3}$ branched along a quartic surface with not only ordinary double points, but other isolated singularities. We also show that if the quartic surface is in the most degenerate form, the twistor space admits a non-trivial $\mathbf{C}^{*}$ action. Correspondingly, $3 \mathbf{C P}^{2}$ admits a self-dual metric of positive scalar curvature with $U(1)$-symmetry, other than LeBrun's famous metrics. This leads us to a classification of twistor spaces over $3 \mathbf{C P}^{2}$ whose automorphism group is non-trivial (or equivalantly, self-dual metrics on $3 \mathbf{C P}^{2}$ whose isometric group is non-trivial).
\end{abstract}

\section{Introduction}

Let $M$ be an oriented four-dimensional manifold and $g$ a Riemannian metric on $M . g$ is said to be self-dual if the anti-self-dual part of the Weyl conformal curvature is identically zero. The famous results of M. Freedman and S.K.Donaldson, and a Bochner type argument imply that if a simply connected compact four-dimensional manifold $M$ admits a self-dual metric of positive scalar curvature, then $M$ must be homeomorphic to the foursphere or $n \mathbf{C P}^{2}$, the connected sum of $n$ copies of the complex projective planes. In his pioneer work Y.S.Poon [18] showed that such a self-dual metric on the four-sphere and the complex projective plane must be the standard ones. Further he explicitly constructed a one-dimensional family of self-dual metrics of positive scalar curvature on $2 \mathbf{C P}^{2}$, and proved that there exists no such metric other than his metrics. Hence the situation is completely understood for the case $n=1$ and 2 .

In this paper we will focus our attention to the case $n=3$. In this case there are works of B.Kreussler [11], Kreussler-H.Kurke [12] and Poon [19]. They studied geometry of the associated twistor space, especially the half of the anticanonical system canonically attached to any twistor spaces. Their results show that the situation is quite different depending on whether the system has base locus or not. Let $\Phi: Z \rightarrow \mathbf{C P}^{r}$ be the meromorphic map 
associated to the system. When the base locus of the system is non-empty, the twistor space $Z$ must be so called LeBrun twistor space [14], and this is also completely understood by works of C. LeBrun [14], Kurke [13] and Poon [19].

On the other hand, when the base locus of the half of the anticanonical system is empty, Kreussler-Kurke [12] and Poon [19] showed that the morphism $\Phi$ is a double covering over $\mathbf{C P}^{3}$ branched along a quartic surface with isolated singularities. This kind of spaces are called double solids. They showed that if the twistor space is sufficiently general, the number of the singular points of the quartic surface is just thirteen and all of them are ordinary double points. (Moreover they obtained defining equation of the quartic surface.) We call these twistor spaces general double solids. Furthermore, Kreussler-Kurke [12] analyzed the situation very carefully and showed that the branched quartic surface may degenerate to have singularities worse than the ordinary double points. We call this kind of twistor spaces degenerate double solids. (See Definition 1.3 for precisely.)

Concerning the existence of these double solids as twistor spaces, Donaldson-R.Friedman [4, p.238, Remark] showed that general double solids exist. (We note that the above results of $[12,19]$ do not show the existence of these spaces as twistor space.) We show that there actually exist twistor spaces (over $3 \mathbf{C P}^{2}$ ) which are degenerate double solids in the above sense. We also study the automorphism groups of these spaces, and determine which double solid has a non-trivial automorphism group. Together with the works of LeBrun [14] and A.Fujiki [5] this completes a classification of twistor spaces over $3 \mathbf{C P}^{2}$ whose automorphism group is non-trivial.

In Section 1 we recall a result of Kreussler-Kurke [12] and give a definition of degenerate double solid (as twistor space) in the title. In Section 2 we prove the existence of degenerate double solids (as twistor space). Theorem 2.1 is the main result of this section. In Section 3 we determine which double solid admits a non-trivial $\mathbf{C}^{*}$-action.

\section{Degenerate double solids as twistor spaces.}

In this section we briefly outline results of Kreussler-Kurke [12] on twistor spaces over $3 \mathbf{C P}^{2}$, and define degenerate double solid as twistor space.

Let $g$ be a self-dual metric on $3 \mathbf{C P}^{2}$ and assume that the sign of the scalar curvature of the conformal class of $g$ is positive. Let $Z$ be the twistor space of $g$ and $\sigma: Z \rightarrow Z$ the real structure (that is, the anti-holomorphic involution without fixed point, naturally defined from the definition of the twistor space) [1]. Let $-K_{Z}$ be the inverse of the canonical bundle (that is, 
the anticanonical bundle) of $Z$. Then $-K_{Z}$ has a natural square root as a holomorphic line bundle, which we denote by $-\frac{1}{2} K_{Z}$.

By Riemann-Roch formula, Serre duality, adjunction formula, and the vanishing theorem of Hitchin [6], we have

$$
\operatorname{dim} H^{0}\left(-\frac{1}{2} K_{Z}\right)-\operatorname{dim} H^{1}\left(-\frac{1}{2} K_{Z}\right)=4 .
$$

In particular, the dimension of the complete linear system $\left|-\frac{1}{2} K_{Z}\right|$ is at least three.

The structure of $Z$ is quite different depending on whether the base locus of $\left|-\frac{1}{2} K_{Z}\right|$ is empty or not. Now assume that $\left|-\frac{1}{2} K_{Z}\right|$ is free from the base locus and let $\Phi: Z \rightarrow \mathbf{C P}^{r}$ be the associated morphism, where $r=\operatorname{dim} H^{0}\left(-\frac{1}{2} K_{Z}\right)-1$. We now see that $r=3$ and $\Phi$ is a double covering branched along a quartic surface.

First since we have $\left(-\frac{1}{2} K_{Z}\right)^{3}=\frac{1}{8} c_{1}(Z)^{3}=2$ [7], the image of $\Phi$ is three dimensional and hence $Z$ is Moishezon. By Bertini's theorem, general member of $\left|-\frac{1}{2} K_{Z}\right|$ is irreducible and smooth. Let $S$ be such a member. Since $Z$ is Moishezon, $S$ must be a projective surface. Since $Z$ is a simply connected twistor space, we have $H^{1}\left(\mathcal{O}_{Z}\right)=0$ [16]. Further, Serre duality and the vanishing theorem of Hitchin imply $H^{2}\left(\frac{1}{2} K_{Z}\right) \simeq H^{1}\left(\frac{1}{2} K_{Z}\right)^{\vee}=0$. Therefore the cohomology exact sequence of

$$
0 \rightarrow \frac{1}{2} K_{Z} \rightarrow \mathcal{O}_{Z} \rightarrow \mathcal{O}_{S} \rightarrow 0
$$

implies that $S$ is a regular surface. Taking tensor product of (1.2) with $\frac{m}{2} K_{Z}$ and using the adjunction formula, we get

$$
0 \rightarrow \frac{m+1}{2} K_{Z} \rightarrow \frac{m}{2} K_{Z} \rightarrow m K_{S} \rightarrow 0 .
$$

From this, we obtain $H^{0}\left(m K_{S}\right)=0$ for any positive $m$. Hence by the classification of surfaces, $S$ must be a rational surface.

The cohomology exact sequence of

$$
0 \rightarrow \mathcal{O}_{Z} \rightarrow-\frac{1}{2} K_{Z} \rightarrow-K_{S} \rightarrow 0
$$

and $H^{1}\left(\mathcal{O}_{Z}\right)=0$ imply that the base locus of $\left|-\frac{1}{2} K_{Z}\right|$ coincides with that of $\left|-K_{S}\right|$. Hence by assumption $\left|-K_{S}\right|$ is also free. Moreover we have $\left(-K_{S}\right)^{2}=\left(-\left.\frac{1}{2} K_{Z}\right|_{S}\right)^{2}=\left(-\frac{1}{2} K_{Z}\right)^{3}=2$. Therefore by Riemann-Roch 
$\left|-K_{S}\right|$ is at least two-dimensional. Then similar argument as above shows that general member of $\left|-K_{S}\right|$ is a smooth elliptic curve. Let $C$ be such a member. Then we have an exact sequence $0 \rightarrow \mathcal{O}_{S} \rightarrow-K_{S} \rightarrow-\left.K_{S}\right|_{C} \rightarrow 0$, from which we get $H^{1}\left(-K_{S}\right)=0$. (Note that $\operatorname{deg}\left(-\left.K_{S}\right|_{C}\right)=2$.) Therefore by the cohomology exact sequence of (1.3), we deduce that $H^{1}\left(-\frac{1}{2} K_{Z}\right)=0$. As a consequence, we get $r=3$ by (1.1) and $\Phi: Z \rightarrow \mathbf{C P}^{3}$ is a double covering map.

Let $R(\subset Z)$ (resp. $\left.B\left(\subset \mathbf{C P}^{3}\right)\right)$ be the ramification divisor (resp. the branch divisor) of $\Phi$. $R$ is a member of $\left|K_{Z} \otimes \Phi^{*} K_{\mathbf{C P}^{3}}^{-1}\right|=$ $\left|K_{Z} \otimes \Phi^{*} \mathcal{O}_{\mathbf{C P}^{3}}(4)\right| . \quad$ But since $-\frac{1}{2} K_{Z}=\Phi^{*} \mathcal{O}_{\mathbf{C P}^{3}}(1)$, this is equal to $\left|\Phi^{*} \mathcal{O}_{\mathrm{CP}^{3}}(2)\right|$. On the other hand since $\Phi$ is a double covering, $R$ is a member of $\left|\frac{1}{2} \Phi^{*} \mathcal{O}_{\mathbf{C P}^{3}}(B)\right|$. From these we see that $B$ is a quartic surface.

Following terminology of Y.S.Poon an elementary divisor means an effective divisor on $Z$ whose intersection number with a twistor line is equal to one. Recall that elementary divisor is always irreducible, and if $D$ is an elementally divisor, $\bar{D}:=\sigma(D)$ is also an elementary divisor and $D+\bar{D}$ is a member of $\left|-\frac{1}{2} K_{Z}\right|$ [19]. The following result of Kreussler and Kurke [12] describe and classify geometric structure of the map $\Phi$. (See also Poon [19].)

Proposition 1.1. Let $g$ be a self-dual metric on $3 \mathbf{C P}^{2}$ with positive scalar curvature and assume that $\left|-\frac{1}{2} K_{Z}\right|$ is free from base locus. Let $\Phi: Z \rightarrow \mathbf{C P}^{3}$ be the double covering induced by $\left|-\frac{1}{2} K_{Z}\right|$, branched along a quartic surface $B$ as above. Then

(i) $\Phi$ does not contract any divisor on $Z$,

(ii) the number of elementary divisors on $Z$ is exactly eight.

Let $\left\{D_{1}, \bar{D}_{1}, \cdots, D_{4}, \bar{D}_{4}\right\}$ be the set of elementary divisors on $Z$, and for $(1 \leq i \leq 4)$, define $w_{i} \in \mathbf{C P}^{3}=\mathbf{P} H^{0}\left(-\frac{1}{2} K_{Z}\right)$ (the space of lines of $\left.H^{0}\left(-\frac{1}{2} K_{Z}\right)\right)$ to be the points naturally determined by the reducible members $D_{i}+\bar{D}_{i} \in\left|-\frac{1}{2} K_{Z}\right|$. Then

(iii) Case (a): if $\left\{w_{1}, w_{2}, w_{3}, w_{4}\right\}$ lies on a line of $\mathbf{C P}^{3}$, then the number of irreducible curves contracted by $\Phi$ is just seven, and the singular locus of $B$ consists of three points. Case (b): If just three of $\left\{w_{1}, w_{2}, w_{3}, w_{4}\right\}$ lies on a line of $\mathbf{C P}^{3}$, then the number of irreducible curves contracted by $\Phi$ is just eleven, and the singular locus of $B$ consists of nine points. Case (c): If any three of $\left\{w_{1}, w_{2}, w_{3}, w_{4}\right\}$ are not on a line, then the number of irreducible curves contracted by $\Phi$ is just thirteen, and the singular locus of $B$ consists of thirteen points, all of which are ordinary double points.

Remark 1.2. $\Phi$ factors through as follows: $\Phi=\Phi_{0} \cdot \alpha$, where $\Phi_{0}: Z_{0} \rightarrow$ 
$\mathbf{C P}^{3}$ is the double covering canonically attached to the branch quartic surface, and $\alpha: Z \rightarrow Z_{0}$ is a small resolution [12, p.44, Lemma 1].

Definition 1.3. Let $Z$ be a twistor space as in Proposition 1.1. We call $Z$ a degenerate double solid of type (a) (resp. of type (b)) if the twistor space $Z$ falls into the case (a) (resp. case (b)) of Proposition 1.1. We call the twistor space $Z$ a general double solid if $Z$ falls into the case (c) of Proposition 1.1.

Concerning the existence of these kinds of twistor spaces, DonaldsonFriedman [4, p.238, Remark] shows that general double solids (defined above) as twistor space actually exist. In the next section we will show that degenerate double solids as twistor spaces also exist for both type (a) and type (b).

\section{Existence of degenerate double solids as twistor spaces.}

In the last section we have assumed that $\left|-\frac{1}{2} K_{Z}\right|$ is free from the base locus. When $\left|-\frac{1}{2} K_{Z}\right|$ has base locus, $Z$ must be [12] so called LeBrun twistor space [14]. LeBrun twistor spaces over $3 \mathbf{C P}^{2}$ are naturally parametrized by three points on the upper half-space $H^{3}$ with standard hyperbolic metric. When the three points are in general position in the sense that they do not lie on a geodesic of $H^{3}$, then LeBrun twistor space has only (effective) $\mathbf{C}^{*}$-action. But if the three points are on a geodesic, the corresponding LeBrun twistor space admits an effective action of $\mathbf{C}^{*} \times \mathbf{C}^{*}$. In the following we call this special LeBrun twistor space LeBrun twistor space with torus action.

The main result of this section is the following

Theorem 2.1. Let $Z_{0}$ be any LeBrun twistor space over $3 \mathbf{C P}^{2}$ with torus action. Then $Z_{0}$ can be deformed into degenerate double solid of type (a) and type (b) (see Definition 1.3) as twistor space. More precisely, there is a proper surjective holomorphic map $p: \mathcal{Z} \rightarrow \Delta \subset \mathbf{C}$ between a complex manifold $\mathcal{Z}$ and an open disk $\Delta \ni 0$ in $\mathbf{C}$ satisfying the following properties: (i) $\mathcal{Z}$ possesses an anti-holomorphic involution $\tilde{\sigma}$ with $p \cdot \tilde{\sigma}=\sigma_{\Delta} \cdot p$, where $\sigma_{\Delta}$ denotes the complex conjugation, (ii) $p^{-1}(0)$ is biholomorphic to $Z_{0}$, (iii) if $t \in \Delta$ is real (i.e. $\sigma_{\Delta}(t)(=\bar{t})=t$ ), then $Z_{t}=p^{-1}(t)$ is a twistor space over $3 \mathbf{C P}^{2}$ which is a degenerate double solid of type (a). The same statement also holds for type (b).

(See Remark 2.8 for $\mathbf{C}^{*}$-equivariant deformations.) Our proof of Theorem 2.1 is outlined as follows:

- First step: We find some $\mathbf{C}^{*} \times \mathbf{C}^{*}$-invariant member $S_{0}$ of $\left|-\frac{1}{2} K_{Z_{0}}\right|$. 
- Second step: We define some special deformation $S_{t}$ of $S_{0}$ such that $\left|-K_{S_{t}}\right|$ is free. (We construct such deformations for each case (a) and (b).)

- Third step: We show that $Z_{0}$ can be deformed into a twistor space $Z_{t}$ which has the above $S_{t}$ as a real member of $\left|-\frac{1}{2} K_{Z_{t}}\right|$.

- Final step: We see that if a twistor space $Z_{t}$ (over $3 \mathbf{C P}^{2}$ ) has $S_{t}$ as a member of $\left|-\frac{1}{2} K_{Z_{t}}\right|,\left|-\frac{1}{2} K_{Z_{t}}\right|$ is free. Then using $S_{t}$ we define some pencil $\mathcal{P}_{t}$ of $\left|-\frac{1}{2} K_{Z_{t}}\right|$ and prove that $\mathcal{P}_{t}$ has just four (resp. just three) reducible members for the case (a) (resp. the case (b)).

For the first step, we consider $\mathbf{C P}^{1} \times \mathbf{C P}^{1}$ with an anti-holomorphic involution $\tau:\left(z_{0}: z_{1}\right) \times\left(w_{0}: w_{1}\right) \mapsto\left(\bar{z}_{0}: \bar{z}_{1}\right) \times\left(\bar{w}_{1}:-\bar{w}_{0}\right)$, where $\left(z_{0}: z_{1}\right)$ and $\left(w_{0}: w_{1}\right)$ are homogeneous coordinates on each $\mathbf{C P}^{1}$. Throughout this section a fiber of the projection onto the first factor $\mathbf{C P}^{1} \times \mathbf{C P}^{1} \rightarrow \mathbf{C P}^{1}$ is a curve of bidegree $(1,0)$. Further we use the following notations: for any point $p$ of $\mathbf{C P}^{1} \times \mathbf{C} \mathbf{P}^{1}, l(p)$ (resp. $\left.l^{\prime}(p)\right)$ denotes the unique curve of bidegree $(1,0)$ (resp. $(0,1))$ going through $p$. We put $p_{1}:=(\sqrt{-1}, 0), \bar{p}_{1}:=$ $\tau\left(p_{1}\right)(=(-\sqrt{-1}, \infty)), p_{2}:=(-\sqrt{-1}, 0)$ and $\bar{p}_{2}:=\tau\left(p_{2}\right)(=(\sqrt{-1}, \infty))$. (Here $0=(1: 0), \infty=(0: 1), \sqrt{-1}=(1: \sqrt{-1})$ and $-\sqrt{-1}=(1:-\sqrt{-1})$.) Further, let $v_{1}$ (resp. $\left.\bar{v}_{1}\right)$ be the tangent vector of $l^{\prime}\left(p_{1}\right)\left(=l^{\prime}\left(p_{2}\right)\right)$ (resp. $\left.l^{\prime}\left(\bar{p}_{1}\right)\left(=l^{\prime}\left(\bar{p}_{2}\right)\right)\right)$ at $p_{1}$ (resp. $\left.\bar{p}_{1}\right)$.

Let $S_{(0)} \rightarrow \mathbf{C P}^{1} \times \mathbf{C P}^{1}$ be the blowing up at $\left\{\left(p_{1}, v_{1}\right),\left(\bar{p}_{1}, \overline{v_{1}}\right), p_{2}, \bar{p}_{2}\right\}$. This implies that we first blow up at four distinct points $\left\{p_{1}, \bar{p}_{1}, p_{2}, \bar{p}_{2}\right\}$ and next blow up at two points which is determined by $v_{1}$ and $\bar{v}_{1}$ on the exceptional curves over $p_{1}$ and $\bar{p}_{1} . S_{(0)}$ is clearly a toric surface with $c_{1}^{2}=2$, and the above $\tau$ naturally lifts to define a real structure of $S_{(0)}$.

Proposition 2.2. $[5,9]$ Let $Z_{0}$ be a LeBrun twistor space over $3 \mathbf{C P}^{2}$ with torus action. Then there exists a $\mathbf{C}^{*} \times \mathbf{C}^{*}$-invariant real irreducible member $S_{0}$ of $\left|-\frac{1}{2} K_{Z_{0}}\right|$ such that there is a biholomorphic map $S_{0} \rightarrow S_{(0)}$ compatible with the real structures.

Proof. It is known [14] that $\left|-\frac{1}{2} K_{Z_{0}}\right|$ is three-dimensional and the base locus consists of disjoint two smooth rational curves. Let $\Phi_{0} \rightarrow \mathbf{C P}^{3}$ be the associated meromorphic map, which is $\mathbf{C}^{*} \times \mathbf{C}^{*}$-invariant. Then the image of $\Phi_{0}$ is a $\mathbf{C}^{*} \times \mathbf{C}^{*}$-invariant non-singular quadric surface. Since $\Phi_{0}$ can also be considered as a quotient map for a $\mathbf{C}^{*}$-action where $\mathbf{C}^{*}$ is certain subgroup of $\mathbf{C}^{*} \times \mathbf{C}^{*}, \mathbf{C}^{*} \times \mathbf{C}^{*}$-action on the quadric reduces to a $\mathbf{C}^{*}$-action. The fixed locus of this $\mathbf{C}^{*}$-action on the quadric consists of two points, which define a pencil of $\mathbf{C}^{*}$-invariant hyperplane sections of the quadric. Then we choose a general real member $H_{0} \cap \Phi_{0}\left(Z_{0}\right)$ of this pencil, and set $S_{0}:=\Phi_{0}^{-1}\left(H_{0}\right)$. Then $S_{0}$ is the desired $\mathbf{C}^{*} \times \mathbf{C}^{*}$-invariant divisor which is biholomorphic to 
$S_{(0)}$. q.e.d.

Next we proceed to the second step. For the type (a), we set $p_{2}(t):=$ $(-i, t)$ ( $t$ is a complex number) and define $S_{t}$ as the surface obtained from $\mathbf{C P}^{1} \times \mathbf{C P}^{1}$ by blowing up $\left\{\left(p_{1}, v_{1}\right),\left(\bar{p}_{1}, \overline{v_{1}}\right), p_{2}(t), \overline{p_{2}(t)}\right\}$.

For the type $(\mathrm{b})$, we set $p_{2}(t):=(-\sqrt{-1}, t), p_{3}(t)=(\sqrt{-1}+t, 0)$ and define $S_{t}$ to be the surface obtained from $\mathbf{C P}^{1} \times \mathbf{C P}^{1}$ by blowing up $\left\{p_{1}, \bar{p}_{1}, p_{2}(t), \overline{p_{2}(t)}, p_{3}(t), \overline{p_{3}(t)}\right\}$.

In both cases, the cycle $l^{\prime}\left(p_{1}\right)+l\left(p_{2}\right)+l^{\prime}\left(\bar{p}_{1}\right)+l\left(\bar{p}_{2}\right)$ on $\mathbf{C P}^{1} \times \mathbf{C P}^{1}$ naturally defines an anti-canonical curve $C_{t}$ of $S_{t}$. If $t \neq 0, C_{t}$ is a cycle of rational curves whose irreducible components are eight (resp. six) for the type (a) (resp. the type (b)).

Lemma 2.3. If $t \neq 0,\left|-K_{S_{t}}\right|$ is free for both cases (a) and (b).

Proof. For the type (a) we show that there are curves $C_{1 t}$ and $\bar{C}_{1 t}$ of $\left|-K_{S_{t}}\right|$ such that $C_{t} \cap C_{1 t} \cap \bar{C}_{1 t}$ is empty. Consider a non-reduced curve $2 l\left(p_{1}\right)+$ $l^{\prime}\left(\bar{p}_{1}\right)+l^{\prime}\left(p_{2}(t)\right)$, which is an anti-canonical curve of $\mathbf{C P}^{1} \times \mathbf{C} \mathbf{P}^{1}$. This curve naturally defines a non-reduced member of $\left|-K_{S_{t}}\right|$. Then from the explicit construction of the surface $S_{t}$, it is easily verified that $C_{t} \cap C_{1 t} \cap \bar{C}_{1 t}=\phi$. This implies that $\left|-K_{S_{t}}\right|$ is free.

For the type (b), because the blown-up points are distinct, it is easily seen that the self-intersection number of any irreducible curve on $S_{t}$ is at least -2 . That is, the configuration of blown up points are in almost general position in the sense of Demazure [3]. Hence $\left|-K_{S_{t}}\right|$ is free. q.e.d.

The third step is not difficult.

Proposition 2.4. Let $Z_{0}$ be any LeBrun twistor space (which is not necessarily with torus action) and $S_{0}$ be any real irreducible member of $\left|-\frac{1}{2} K_{Z_{0}}\right|$. Then $S_{0}$ is costable in $Z_{0}$. That is, for any deformation $q: \mathcal{S} \rightarrow B \subset \mathbf{C}^{n}$ with $q^{-1}(0) \simeq S_{0}$, there is a deformation $p: \mathcal{Z} \rightarrow B^{\prime} \subset B$ and an embedding $i: \mathcal{S} \rightarrow \mathcal{Z}$ such that $q=p \cdot i$ over $B^{\prime}$.

Proof. By a theorem of Horikawa[10], it suffices to prove that $H^{2}\left(\Theta_{Z_{0}}\left(-S_{0}\right)\right)=0$. But this follows from Lemma 1.9 of Campana[2] and a result of Kreussler[11], since $Z_{0}$ is Moishezon. q.e.d.

To complete our proof of Theorem 2.1, we proceed to the final step. Let $q: \mathcal{S} \rightarrow \Delta \subset \mathbf{C}$ be the one-dimensional family of deformation of $S_{0}$ constructed in the second step, and let $p: \mathcal{Z} \rightarrow \Delta^{\prime} \subset \mathbf{C}, 0 \in \Delta^{\prime} \subset \Delta$ 
with $i: \mathcal{S} \rightarrow \mathcal{Z}$ be a family of deformation of $Z_{0}$ whose existence is guaranteed by Proposition 2.4. Recall that if $t \neq 0$, there is a real anticanonical curve $C_{t}$ of $S_{t}$ which consists of eight (for the case (a)) or six (for the case (b)) rational curves. Since $S_{t}$ is a member of $\left|-\frac{1}{2} K_{Z_{t}}\right|$, we have an exact sequence $0 \rightarrow \mathcal{O}_{Z_{t}} \rightarrow-\frac{1}{2} K_{Z_{t}} \rightarrow-K_{S_{t}} \rightarrow 0$. This induces an exact sequence $0 \rightarrow H^{0}\left(\mathcal{O}_{Z_{t}}\right) \rightarrow H^{0}\left(-\frac{1}{2} K_{Z_{t}}\right) \rightarrow H^{0}\left(-K_{S_{t}}\right) \rightarrow 0$. By Lemma 2.3, $\left|-K_{S_{t}}\right|$ is free. Hence the surjectivity of the above restriction map $r: H^{0}\left(-\frac{1}{2} K_{Z_{t}}\right) \rightarrow H^{0}\left(-K_{S_{t}}\right) \rightarrow 0$ implies that $\left|-\frac{1}{2} K_{Z_{t}}\right|$ is also free. Let $L_{t} \subset H^{0}\left(-K_{S_{t}}\right)\left(=\mathbf{C}^{3}\right)$ be the one-dimensional subspace generated by a section defining the above $C_{t}$, and set $V_{t}:=r^{-1}\left(L_{t}\right) . V_{t}$ is a two-dimensional subspace of $H^{0}\left(-\frac{1}{2} K_{Z_{t}}\right)$, and invariant under the complex conjugation induced by the real structure of $Z_{t}$, since both $S_{t}$ and $C_{t}$ are real. Let $\mathcal{P}_{t} \subset\left|-\frac{1}{2} K_{Z_{t}}\right|$ be the pencil determined by $V_{t}$.

The following proposition completes our proof of Theorem 2.1.

Proposition 2.5. For the case (a), $\mathcal{P}_{t}$ has just four reducible members, and for the case (b), just three reducible members.

To prove this, we need some preliminaries. The first is a result of Pedersen-Poon[17] stating that for any twistor space $Z$ over $n \mathbf{C P}^{2}$, any irreducible real member $S$ of $\left|-\frac{1}{2} K_{Z}\right|$ is obtained from $\mathbf{C P}^{1} \times \mathbf{C P}^{1}$ by blowing up $n$ conjugates pairs of points. Once we fix a blowing down $\mu: S \rightarrow \mathbf{C P}^{1} \times \mathbf{C P}^{1}$, an orthonormal basis of $H^{2}\left(n \mathbf{C P}^{2}, \mathbf{Z}\right)$ is naturally defined as follows: let $\left\{E_{1}, E_{2}, \cdots E_{n}, \bar{E}_{1}, \bar{E}_{2}, \cdots \bar{E}_{n}\right\}$ be the set of exceptional curves of $\mu$ satisfying $E_{i} \cdot E_{j}=\bar{E}_{i} \cdot \bar{E}_{j}=-\delta_{i j}$ (Kronecker delta) and $E_{i} \cdot \bar{E}_{j}=0$ for any $1 \leq i, j \leq n$. Let $\pi: Z \rightarrow n \mathbf{C P}^{2}$ be the twistor fibration. Since $\left.\pi\right|_{S}: S \rightarrow n \mathbf{C P}^{2}$ is an orientation preserving diffeomorphism on some connected neighborhood of $\left\{E_{1}, E_{2}, \cdots E_{n}\right\}$, this set of curves determines an orthonormal basis of $H^{2}\left(n \mathbf{C P}^{2}, \mathbf{Z}\right)$. We denote these by $\left\{\alpha_{1}, \alpha_{2}, \cdots, \alpha_{n}\right\}$. Then the following two lemmas proved in the proof of Proposition 1.2 in [8, pp. 649-650] are crucial:

Lemma 2.6. Let $\pi: Z \rightarrow n \mathbf{C P}^{2}$ be any twistor space and $S$ any real irreducible (hence smooth) member of $\left|-\frac{1}{2} K_{Z}\right|$. Fix a blowing down $\mu: S \rightarrow$ $\mathbf{C P}^{1} \times \mathbf{C P}^{1}$ as above and let $\left\{E_{1}, \cdots E_{n}, E_{1}, \cdots \bar{E}_{n}\right\}$ be the set of exceptional curves, and $\left\{\alpha_{1}, \cdots, \alpha_{n}\right\}$ the induced orthonormal basis of $H^{2}\left(n \mathbf{C P}^{2}, \mathbf{Z}\right)$. Let $\sigma_{1}, \cdots, \sigma_{n}$ be odd integers and

$$
\mathcal{D}_{\sigma_{1} \cdots \sigma_{n}}=\frac{1}{4} c_{1}(Z)+\frac{1}{2} \sum_{i=1}^{n} \sigma_{i}\left(\pi^{*} \alpha_{i}\right)
$$


an element of $H^{2}(Z, \mathbf{Z})$. Then as elements of $H^{2}(S, \mathbf{Z})$, we have

$$
\left.\mathcal{D}_{\sigma_{1} \cdots \sigma_{n}}\right|_{S}=\mu^{*} \mathcal{O}(1,1)-\frac{1}{2} \sum_{i=1}^{n}\left(1-\sigma_{i}\right) E_{i}-\frac{1}{2} \sum_{i=1}^{n}\left(1+\sigma_{i}\right) \bar{E}_{i} .
$$

Lemma 2.7. Let $\mathcal{D}_{\sigma_{1} \cdots \sigma_{n}} \in H^{2}(Z, \mathbf{Z})$ be as above. If $\sigma_{k}= \pm 1$ for any $1 \leq$ $k \leq n$, then the natural restriction map $H^{i}\left(Z, \mathcal{D}_{\sigma_{1} \cdots \sigma_{n}}\right) \rightarrow H^{i}\left(S,\left.\mathcal{D}_{\sigma_{1} \cdots \sigma_{n}}\right|_{S}\right)$ is an isomorphism for any $i \geq 0$. In particular, if $\left|\mathcal{D}_{\sigma_{1} \cdots \sigma_{n}}\right| S \mid$ is non-empty, then $\left|\mathcal{D}_{\sigma_{1} \cdots \sigma_{n}}\right|$ is also non-empty.

Proof of Proposition 2.5. We only prove the claim for the case (a). Case (b) can be proved in the same way.

By the construction explained after Proposition 2.4, we are in the following situation: $Z_{t}$ is a twistor space over $3 \mathbf{C P}^{2}, S_{t}$ is a real smooth member of $\left|-\frac{1}{2} K_{Z_{t}}\right|$, and there is a real anticanonical curve $C_{t}$ of $S_{t}$ consisting of eight rational curves. We may write $C_{t}=\sum_{i=1}^{4} C_{i}+\sum_{i=1}^{4} \bar{C}_{i}$, where $C_{i}$ and $\bar{C}_{i}=\sigma\left(C_{i}\right)$ are smooth rational curves satisfying $C_{1} \cdot C_{2}=C_{2} \cdot C_{3}=$ $C_{3} \cdot C_{4}=C_{4} \cdot \bar{C}_{1}=\bar{C}_{1} \cdot \bar{C}_{2}=\bar{C}_{2} \cdot \bar{C}_{3}=\bar{C}_{3} \cdot \bar{C}_{4}=\bar{C}_{4} \cdot C_{1}=1$ and $C_{i} \cdot C_{j}=C_{i} \cdot \bar{C}_{j}=0$ for the remaining $\{i, j\}$ with $i \neq j$. From the explicit construction of $S_{t} \rightarrow \mathbf{C P}^{1} \times \mathbf{C P}^{1}$, we can assume that $C_{1}^{2}=\bar{C}_{1}^{2}=-1$ and $C_{i}^{2}=\bar{C}_{i}^{2}=-2$ for $i=2,3,4$. Further, as homology classes, we have

$$
\begin{gathered}
C_{1}=E_{3}, \bar{C}_{1}=\bar{E}_{3}, \\
C_{2}=(1,0)-E_{1}-E_{3}, \bar{C}_{2}=(1,0)-\bar{E}_{1}-\bar{E}_{3}, \\
C_{3}=(0,1)-\bar{E}_{1}-E_{2}, \bar{C}_{3}=(0,1)-E_{1}-\bar{E}_{2},
\end{gathered}
$$

and

$$
C_{4}=\bar{E}_{1}-\bar{E}_{3}, \bar{C}_{4}=E_{1}-E_{3} .
$$

(Here we denote $(a, b):=\mu^{*} \mathcal{O}(a, b)$.) From these we obtain

$$
\begin{aligned}
& C_{1}+C_{2}+C_{3}+C_{4}=(1,1)-E_{1}-E_{2}-\bar{E}_{3}, \\
& C_{2}+C_{3}+C_{4}+\bar{C}_{1}=(1,1)-E_{1}-E_{2}-E_{3}, \\
& C_{3}+C_{4}+\bar{C}_{1}+\bar{C}_{2}=(1,1)-\bar{E}_{1}-E_{2}-\bar{E}_{3}, \\
& C_{4}+\bar{C}_{1}+\bar{C}_{2}+\bar{C}_{3}=(1,1)-E_{1}-\bar{E}_{2}-\bar{E}_{3}
\end{aligned}
$$

and the conjugations of these. On the other hand, by Lemma 2.6, we have

$$
\left.\mathcal{D}_{111}\right|_{S}=(1,1)-\bar{E}_{1}-\bar{E}_{2}-\bar{E}_{3},
$$


and

$$
\left.\mathcal{D}_{-1-1-1}\right|_{S}=(1,1)-E_{1}-E_{2}-E_{3} .
$$

Hence $\bar{C}_{2}+\bar{C}_{3}+\bar{C}_{4}+C_{1}$ (resp. $C_{2}+C_{3}+C_{4}+\bar{C}_{1}$ ) is a member of $\left|\mathcal{D}_{111}\right|_{S} \mid$ (resp. $\left.\left|\mathcal{D}_{-1-1-1}\right| S \mid\right)$. Therefore by Lemma $2.7,\left|\mathcal{D}_{111}\right|$ (resp. $\left.\left|\mathcal{D}_{-1-1-1}\right|\right)$ has a member $D_{1}$ (resp. $\bar{D}_{1}$ ) whose restriction on $S$ is $\bar{C}_{2}+\bar{C}_{3}+\bar{C}_{4}+C_{1}$ (resp. $C_{2}+C_{3}+C_{4}+\bar{C}_{1}$ ). In the same way, we can show that if $\sigma_{i}= \pm 1$ for any $i=1,2,3,\left|\mathcal{D}_{\sigma_{1} \sigma_{2} \sigma_{3}}\right|$ has a member such that its restriction on $S$ is just a 'half' of $C_{t}$.

Now we get just eight elementary divisors $\left\{D_{1}, \bar{D}_{1}, \cdots, D_{4}, \bar{D}_{4}\right\}$. To finish our proof of Proposition 2.5, we only need to show that $D_{i}+\bar{D}_{i}$ is a member of the pencil $\mathcal{P}_{t}$ for any $1 \leq i \leq 4$. But this is evident because we know that

$$
\left.\left(D_{i}+\bar{D}_{i}\right)\right|_{S}=\left.D_{i}\right|_{S}+\left.\bar{D}_{i}\right|_{S}=C_{t}
$$

(not only set theoretically, but also scheme theoretically). q.e.d.

Remark 2.8. We make some remarks on the case (a). It is easily seen that our family $q: \mathcal{S} \rightarrow \Delta \subset \mathbf{C}$ explicitly constructed gives a $\mathbf{C}^{*}$-equivariant deformations of $S_{0}$ for the case (a), where $\mathbf{C}^{*}$ is some subgroup of $\mathbf{C}^{*} \times$ $\mathbf{C}^{*}$. Further, we can show that Proposition 2.4 is valid for $\mathbf{C}^{*}$-equivariant deformations. Hence we can strengthen the statement of Theorem 2.1 to the effect that we can $\mathbf{C}^{*}$-equivariantly deform LeBrun twistor space with torus action to get a degenerate double solid of type (a) with a $\mathbf{C}^{*}$-action.

\section{Characterization of degenerate double solid as twistor spaces which has $\mathrm{C}^{*}$-action.}

In Section 1 we have seen that a twistor space over $3 \mathbf{C P}^{2}$ whose system $\left|-\frac{1}{2} K_{Z}\right|$ is free must be a double covering of $\mathbf{C P}^{3}$ branched over a quartic surface, and explained the result (Proposition 1.1) of Kreussler-Kurke [12] which says that such twistor spaces can be classified into the case (a), (b) and (c), according to the configuration of reducible members, or the number of singular points of the branch quartic surface. In this section, among these spaces, we characterize twistor spaces $Z$ which admit a non-trivial $\mathbf{C}^{*}$-action. That is, we will show the following

Proposition 3.1. Let $Z$ be a twistor space over $3 \mathbf{C P}^{2}$ in Proposition 1.1. Then $Z$ admits an effective $\mathbf{C}^{*}$-action if and only if $Z$ is of type (a). 
Before proving, we give a remark. For a compact complex manifolds $X$, we denote by $\operatorname{Aut}_{0}(X)$ the identity component of the holomorphic transformation group of $X$. It is known that for any twistor space $Z$ over $n \mathbf{C P}^{2}(n \geq 2) \operatorname{Aut}_{0}(Z)$ is either $\mathbf{C}^{*} \times \mathbf{C}^{*}$ or $\mathbf{C}^{*}$, and that if $\operatorname{Aut}_{0}(Z)$ is $\mathbf{C}^{*} \times \mathbf{C}^{*}$, then $Z$ must be a LeBrun twistor space with torus action [5]. Further, as explained in Section 1 , if $\left|-\frac{1}{2} K_{Z}\right|$ has base points, then $Z$ must be LeBrun twistor space, and general LeBrun twistor space satisfies $\operatorname{Aut}_{0}(Z)=\mathbf{C}^{*}$. Hence Proposition 3.1 gives the following:

Corollary 3.2. Let $g$ be a self-dual metric on $3 \mathbf{C P}^{2}$ with positive scalar curvature, and $Z$ the associated twistor space. Then $\operatorname{Aut}_{0}(Z)=\mathbf{C}^{*}$ if and only if $Z$ is LeBrun twistor space without torus action or degenerate double solid of type (a).

Proof of Proposition 3.1. First we show that $Z$ belonging to type (a) admits a $\mathbf{C}^{*}$-action. Let $\Phi: Z \rightarrow \mathbf{C P}^{3}$ be the double covering induced by $\left|-\frac{1}{2} K_{Z}\right|$, which is branched along a quartic surface $B$. By a result of Kreussler-Kurke $[12$, p. 49-50], the equation of $B$ is given by

$$
F=\left(x_{2}^{2}+x_{3}^{2}+Q_{0}\left(x_{0}, x_{1}\right)\right)^{2}-x_{0} x_{1}\left(x_{0}+x_{1}\right) L\left(x_{0}, x_{1}\right),
$$

where $\left(x_{0}: x_{1}: x_{2}: x_{3}\right)$ is homogeneous coordinates of $\mathbf{C P}^{3}, Q_{0}\left(x_{0}, x_{1}\right)$ is a quadratic homogeneous polynomial of $x_{0}$ and $x_{1}$, and $L\left(x_{0}, x_{1}\right)$ is a linear form of $x_{0}$ and $x_{1}$. If we change coordinate as

$$
y_{0}=x_{0}, y_{1}=x_{1}, y_{2}=x_{2}+\sqrt{-1} x_{3}, y_{3}=x_{2}-\sqrt{-1} x_{3}
$$

then the equation becomes

$$
F=\left(y_{2} y_{3}+Q_{0}\left(y_{0}, y_{1}\right)\right)^{2}-y_{0} y_{1}\left(y_{0}+y_{1}\right) L\left(y_{0}, y_{1}\right) .
$$

Then a $\mathbf{C}^{*}$-action given by $\left(y_{0}: y_{1}: y_{2}: y_{3}\right) \mapsto\left(y_{0}: y_{1}: t y_{2}: t^{-1} y_{3}\right)$ for $t \in \mathbf{C}^{*}$ preserves the above equation invariant and hence naturally induces a $\mathbf{C}^{*}$-action on $Z$. (Here, the singular locus of $B$ consists of three points $\left(y_{0}: y_{1}: y_{2}: y_{3}\right)=(0: 0: 0: 1),(0: 0: 1: 0)$ and the double root of the equation $Q_{0}\left(y_{0}, y_{1}\right)^{2}+y_{0} y_{1}\left(y_{0}+y_{1}\right) L\left(y_{0}, y_{1}\right)=0$. The first two points are conjugate each other, and the last point is real. The pencil $\mathcal{P}_{t}$ (see Section 2) corresponds to the pencil of planes generated by $y_{0}$ and $y_{1}$, and reducible members of $\mathcal{P}_{t}$ correspond to the four planes $y_{0}=0, y_{1}=0, y_{0}+y_{1}=0$, and $L\left(y_{0}, y_{1}\right)=0$. Further, the line $\left\{y_{2}=y_{3}=0\right\}$ is pointwise-fixed. This is the image $\Phi\left(L_{0}\right)$, where $L_{0}$ is the unique twistor line which is pointwise fixed.) 
Next we show that $Z$ of type (c) does not admit $\mathbf{C}^{*}$-action. In this case, the branch surface $B$ is a quartic surface with only ordinary double points [12, pp.50-51]. Hence any minimal resolution of $B$ must be a K3-surface, which does not have continuous symmetry. Therefore the claim follows.

Finally we show that $Z$ of type (b) also does not admit $\mathbf{C}^{*}$-action. Let $D_{1}+\bar{D}_{1}, D_{2}+\bar{D}_{2}$ and $D_{3}+\bar{D}_{3}$ be the reducible members of $\left|-\frac{1}{2} K_{Z}\right|$ belonging to a pencil, and $w_{1}, w_{2}$ and $w_{3}$ be the corresponding points of $\mathbf{P} H^{0}\left(-\frac{1}{2} K_{Z}\right)$, which are on a line $l$. Aut $t_{0}(Z)$ acts naturally on $\mathbf{P} H^{0}\left(-\frac{1}{2} K_{Z}\right)$ and preserves $l$. Further, since $w_{1}, w_{2}$ and $w_{3}$ must be fixed, the action on $l$ must be trivial. Hence $\operatorname{Aut}_{0}(Z)$ is at most $\mathbf{C}^{*}$. However, $\operatorname{Aut}_{0}(Z)$ must also fix $w_{4}$ which corresponds to the remaining reducible member of $\left|-\frac{1}{2} K_{Z}\right|$. By definition of type (b), $w_{4}$ is not on $l$. Therefore $\operatorname{Aut}_{0}(Z)$ must be trivial. q.e.d.

Remark 3.3. Let $Z$ be a twistor space over $3 \mathbf{C P}^{2}$ which is a degenerate double solid of type (a), and $\rho$ the $\mathbf{C}^{*}$-action in Proposition 3.1. It can be shown that $\rho$ is obtained as the complexification of a $U(1)$-action on $Z$, where the $U(1)$-action is induced from $U(1)$-isometry of the corresponding self-dual metric on $3 \mathbf{C P}^{2}$. This $U(1)$-action on $3 \mathbf{C P}^{2}$ is not semi-free, as a characterization theorem of LeBrun [15] implies.

Acknowledgement The author would like to express his gratitude to Professor B.Kreussler for suggestions and encouragement. This work is partially supported by Research Fellowships of the Japan Society for the Promotion of Science for Young Scientist.

\section{References.}

[1] M. Atiyah, N. Hitchin, I. Singer, Self-duality in four dimensional Riemannian geometry, Proc. Roy. Soc. London, Ser. A 362 (1978), 425-461.

[2] F. Campana, The class $\mathcal{C}$ is not stable by small deformations, Math. Ann. 290 (1991), 19-30.

[3] M. Demazure, Surfaces de Del Pezzo - III, IV, Séminaire surles singularité des surfaces, Lecture Notes in Math. 777 (1980).

[4] S. K. Donaldson, R. Friedman, Connected sums of self-dual manifolds and deformations of singular spaces, Nonlinearlity 2 (1989), 197-239. 
[5] A. Fujiki, Compact self-dual manifolds with torus actions, J. Diff. Geom. 55 (2000), 229-324.

[6] N. Hitchin, Linear field equations on self-dual spaces, Proc. London Math. Soc. 370 (1980), 173-191.

[7] N. Hitchin, Kählarian twistor spaces, Proc. London Math. Soc. (3) 43 (1981), 133-150.

[8] N. Honda, Donaldson-Friedman construction and deformations of a triple of compact complex spaces, Osaka J. Math. 36 (1999) 641-672.

[9] N. Honda, On the structure of Pedersen-Poon twistor spaces, to appear in Math. Scand.

[10] E. Horikawa, Deformations of holomorphic maps. III, Math. Ann. 222 (1976), 275-282

[11] B. Kreussler, Small resolutions of double solids, branched over a 13nodal quartic surfaces, Ann. Global Anal. Geom. 7 (1989), 227-267.

[12] B. Kreussler, H. Kurke, Twistor spaces over the connected sum of 3 projective planes, Compositio Math. 82 (1992), 25-55.

[13] H. Kurke, Classification of twistor spaces with a pencil of surfaces of degree 1. I, Math. Nachr. 158 (1992), 67-85.

[14] C. LeBrun, Explicit self-dual metrics on $\mathbf{C P}^{2} \# \cdots \# \mathbf{C P}^{2}$, J. Diff. Geom. 34 (1991), 223-253.

[15] C. LeBrun, Self-dual manifolds and hyperbolic geometry, in: Einstein Manifolds and Yang-Mills Connections (1993), 99-131, Mercel Dekker,

[16] C. LeBrun, Y. Poon, Twistors, Kähler manifolds and bimeromorphic geometry. II, J. Amer. Math.Soc. 5 (1992), 317-325.

[17] H. Pedersen, Y. S. Poon, Self-duality and differentiable structures on the connected sum of complex projective planes, Proc. Amer. Math. Soc. 121 (1994), 859-864.

[18] Y. S. Poon, Compact self-dual manifolds of positive scalar curvature, J. Diff. Geom. 24 (1986), 97-132. 
[19] Y. S. Poon, On the algebraic structure of twistor spaces, J. Diff. Geom. 36 (1992), 451-491.

Department of Mathmatics

Graduate School of Science

HiROSHIMA UNIVERSITY

HigASHI HiRoshima, 739-8526, JAPAN

E-mail: honda@math.sci.hiroshima-u.ac.jp

RECEIVED JANUARY 16, 2001. 